\title{
Investigation of the effects of cavitation on near nozzle dynamics in multi-hole gasoline direct injection sprays
}

\author{
Paul McGinn*1, Alex Gander ${ }^{1}$, Giovanni Tretola ${ }^{1}$, Cyril Crua ${ }^{1}$, Konstantina Vogiatzaki ${ }^{1}$ \\ ${ }^{1}$ Advanced Engineering Centre, University of Brighton, UK \\ ${ }^{*}$ Corresponding author email: p.mcginn2@brighton.ac.uk
}

\begin{abstract}
The accurate control of the the spray dynamics arising from the nozzle in GDI injectors is key in order to control the subsequent combustion process. In this work we present LES of in nozzle dynamics of gasoline direct injection and the resulting early development of spray plumes from an 8-hole injector (ECN Spray G). In order to do so a new three fluid solver within OpenFOAM has been developed. The objective is to evaluate the impact of the cavitation in a multihole gasoline injector, along with its influence on the flow field at the start of injection. It is shown how the interaction between the gaseous phases can influence the initial stages of the spray formation and the different pattern of cavitation across the injectors holes.
\end{abstract}

\section{Keywords}

Cavitation, Multiphase, Sprays, OpenFOAM

\section{Introduction}

The use of gasoline direct injection (GDI) in modern light and heavy-duty vehicles has increased significantly over the past decade. The uptake in GDI technology has been driven by a global push to reach net-zero carbon emissions. A recent study highlighted that hybridised internal combustion engines (ICE) could meet this target using net-zero carbon, liquid fuels that are currently in development [1]. GDI engines as a platform for these net-zero carbon fuels have a requirement of a high level of control on the fuel injection and fuel-air mixing events to achieve the increased efficiencies required for a significant reduction in pollutant emissions [2]. Thus, part of the challenges of further improving these systems are related to understanding the dynamics taking place in and near nozzle regions. In general gasoline sprays operate under lower injection pressures and have lower exit velocities which makes the atomisation harder. Usually, GDI sprays are subjected to injection pressures of 10-40 MPa with an average exit velocity of 200-300 m/s. Moreover, GDI injectors can present phenomena such as flash boiling, cavitation and spray collapse that are significantly different to the better documented diesel sprays.

Overall it is extremely challenging to develop a comprehensive understanding of near-nozzle spray breakup only through experimental techniques because of optical limitations (especially within the nozzle) and resolution issues. Computational Fluid Dynamics (CFD) is a potential complementary tool that can be used to enhance our underlying of the physics of spray atomisation and how fuel/air mixture formation is linked to in nozzle phenomena [3, 4].

The large interest in developing such systems has motivated the Engine Combustion Network (ECN) group to start a topic, focusing on an 8-orifice (stepped-hole) valve covered orifice injectors, built by Delphi for this purpose [5]. The reference operating point for this system is referred as Spray G. Spray G is a suitable validation test case for models that represent the key physics in gasoline sprays. It has the necessary geometrical complexity in order to allow for interacting spray jets dynamics to be explored and it is mildly cavitating which allows to also examine phase change phenomena present in multi-hole injectors.

Key aspects of the modelling of this injector have been previously explored [4, 6, 7, 8, 9], such as the impact of in-nozzle geometry and of the transient needle lift. Flash-boiling effects have 
also be investigated both experimentally $[6,10,11,12]$ and numerically $[4,7,13]$. Experimental findings identify internal nozzle cavitation but are unable to highlight its influence on spray break-up. Two phase modelling has been used previously to explore the effects of cavitation $[3,14]$. Such models isolate and visualise the occurrence of cavitation however they are not directly coupled with the spray propagation.

Here we extend our study in order to include the effect of in nozzle phenomena taking place in multi-hole injectors employing a novel three-fluid (including air,liquid and vapour) solver within OpenFOAM. In the next section, a brief description of the methodology used is presented, followed by the description of the experimental and numerical set-up, then the results are presented. The focus in the presentation of the results is on the cavitation formation in a multi-hole non symmetric geometry. Moreover, imposing an inlet condition constant over the time, the influence of a non symmetric geometry is isolated. In the end the major conclusions will be presented along with some discussion of what the next steps in future work will be.

\section{Methodology}

To investigate the presence and influence of cavitation for the near nozzle behaviour, in our work, a new three fluid solver within OpenFOAM [15] has been developed. The basis of the solver is the Volume of Fluid (VoF) methodology for the three different fluids (the liquid, vapour and nitrogen phases). An artificial compressive velocity term is applied to sharpen the interface between the liquid and the two gaseous phases. Diffusive mixing between the vapour and nitrogen is achieved by including Laplacian diffusion terms in the transport of these two phases. The gaseous nitrogen phase is assumed to not condensate, leaving the phase change occurring only between the liquid and vapour phase. The phase change is modelled as source terms in the transport equation of such phases. This approach allows us to to solve simultaneously the internal nozzle flow and the external spray formation. In comparison to previous works (see for example [9]) modelling the effect of the in nozzle flow phase change and its effect on the near field is also accounted for here.

To account for a three fluid system, the transport of each fluid volume fraction needs to be considered. The transport equation for each fluid can be defined as follows:

$$
\left\{\begin{array}{l}
\frac{\partial \alpha_{l}}{\partial t}+\nabla \cdot\left(\alpha_{l} U\right)+\nabla \cdot\left(\alpha_{l} \alpha_{v}\left(U_{l}-U_{v}\right)+\alpha_{l} \alpha_{n c}\left(U_{l}-U_{n c}\right)\right)=+\dot{m}\left(\frac{1}{\rho_{l}}-\alpha_{l}\left(\frac{1}{\rho_{l}}-\frac{1}{\rho_{v}}\right)\right) \\
\frac{\partial \alpha_{v}}{\partial t}+\nabla \cdot\left(\alpha_{v} U\right)+\nabla \cdot\left(\alpha_{v} \alpha_{l}\left(U_{v}-U_{l}\right)\right)=\nabla \cdot\left(\Gamma \nabla \alpha_{v}\right)-\dot{m}\left(\frac{1}{\rho_{v}}-\alpha_{v}\left(\frac{1}{\rho_{l}}-\frac{1}{\rho_{v}}\right)\right) \\
\frac{\partial \alpha_{n c}}{\partial t}+\nabla \cdot\left(\alpha_{n c} U\right)+\nabla \cdot\left(\alpha_{n c} \alpha_{l}\left(U_{n c}-U_{l}\right)\right)=\nabla \cdot\left(\Gamma \nabla \alpha_{n c}\right)
\end{array}\right.
$$

The subscripts of $\mathrm{l}, \mathrm{v}$ and i denote the liquid, vapour and atmospheric gas phases respectively. $\mathrm{U}$ is the systems velocity field, $\alpha$ is the phase volume fraction, and $\Gamma$ is the diffusion coefficient. $\dot{\mathrm{m}}$ is the volumetric mass transport source term which needs to be dynamically modelled. The Schnerr Sauer cavitation model is derived from the growth of a bubble radius in a transient pressure field and is chosen for this case [16]. The original model considers only two phases thus needs slight modification to account for three:

$$
\begin{aligned}
& \dot{m}_{-}=C_{v} \frac{3 \rho_{1} \rho_{2}}{\rho_{m}}\left(1-\alpha_{l}-\alpha_{i}\right) R_{b} \sqrt{\frac{2 p_{s a t}-p}{3 \rho_{1}}} \\
& \dot{m}_{+}=C_{c} \frac{3 \rho_{1} \rho_{2}}{\rho_{m}}\left(1-\alpha_{l}-\alpha_{i}\right) R_{b} \sqrt{\frac{2 p-p_{s a t}}{3 \rho_{1}}}
\end{aligned}
$$


a)

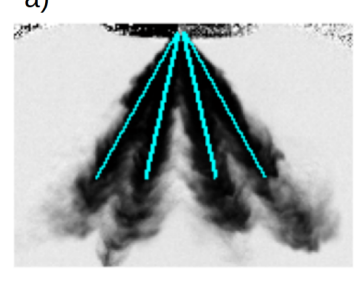

b)

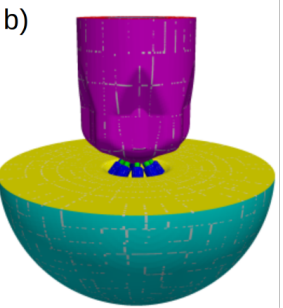

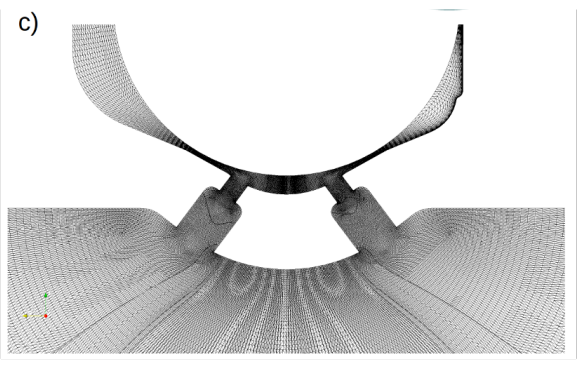

Figure 1. Examples of a) experimental spray formation [18], b) the computational domain, and c) the detail of the mesh near the injector nozzles

\section{Spray G experimental and numerical Set-up}

The experimental set-up consists of an eight-hole counter-bore GDI fuel injector, denoted as the Spray G nozzle in the Engine Combustion Network [5]. The nozzle geometry and spray conditions were determined from the selected ECN experimental test case. The spray system consists of an 8-hole nozzle, with a fuel injection pressure of 200 bar and chamber pressure at 6 bar. The injected mass is $10 \mathrm{mg}$ over $780 \mu \mathrm{s}$. The diameter of each nozzle is $170 \mu \mathrm{m}$ (with an uncertainty of $1.8 \mu \mathrm{m}$ ). The internal geometry is not symmetric, and differences in the flow patterns are expected across the holes, that create different discharge coefficients. The fuel is taken as iso-octane at a temperature of $363 \mathrm{~K}$ while the chamber pressure is $537 \mathrm{~K}$. The computational domain is based on the ECN setup of the in-nozzle geometry and a $3 \mathrm{~mm}$ domain extent, or cap. The needle is set at the fully open position with a gap of $50 \mu \mathrm{m}$. To represent the experimental injection conditions, a pressure difference is imposed between the inlet and outlet boundaries. This set-up has been used previously to model Spray-G, with good agreement on spray penetration [9]. The simulation time is approximately $10 \mu \mathrm{m}$ after the fluid exits the nozzle. This time is short enough so effects of the needle wobble and overshoot can be ignored.

Table 1. Transport properties for each phase, values were obtained from the NIST database taken at the reported temperatures [17]

\begin{tabular}{|c|c|c|c|}
\hline \multicolumn{4}{|c|}{ temperatures [1T] } \\
\hline & Iso-octane & Vapour & Nitrogen \\
\hline Density $\left(\mathrm{kg} / \mathrm{m}^{3}\right)$ & 669.06 & 1.3 & 3.5232 \\
\hline Viscosity $\left(\mathrm{mm}^{2} / \mathrm{s}\right)$ & 49.788 & 5.3038 & 8.1514 \\
\hline Saturation Vap. Pres. (kPa) & 70.9 & - & - \\
\hline Surface Tension $\left(\mathrm{kg} / \mathrm{s}^{2}\right)$ & 0.002 & - & - \\
\hline
\end{tabular}

A conventional curvilinear structured mesh provided by the ECN Workshop [5] and generated with GridPro was used to carry out the simulations. The mesh and domain are shown in Figure 1 along with the spray formation taken from experimental data [18]. The mesh consists of 5.5 million cells, with a minimum size of $5 \mu \mathrm{m}$ in the needle tip and nozzle and an average size of $10 \mu \mathrm{m}$ in the counter-bore. The maximum cell size is $65 \mu \mathrm{m}$ approaching the domain exit. The Taylor length scale can be estimated (for singe phase flow) $\lambda / \mathrm{D} \approx \sqrt{10} \mathrm{Re}^{1 / 2}$, which gives an estimate at the nozzle exit of $1.2 \mu \mathrm{m}$. The mesh-to-Taylor lengths scale ratios of 4-5, which suggests that while the mesh is far from DNS resolution, the resolution is adequate for LES of jet flows. The mesh employed has sufficient resolution to capture the initial shear layer at the nozzle exit.

Cavitation is characterised by non-linear behaviour and is dependent upon flow geometry and solid surface conditions as well as local pressure conditions. Cloud break-up is further augmented by the collapse of previously shed cavities. As such the modelling of cavitation dynamics is sensitive to the numerical discretisation used. A previous study has shown that common 
second order schemes will tend to dampen local large pressure gradients weakening the interaction between initial cloud break-off with previously shed cavities [19]. A higher order TVD scheme called MUSCL was shown to be able to maintain these local pressure gradients and produced a more physically consistent cavitation cloud topology. This discretisation scheme was used together with a PISO algorithm to solve the pressure-momentum coupling. A WALE turbulence model [20] determines the sub-grid viscosity $\nu_{\mathrm{sgs}}$, required for the sub-grid stresses and turbulent transport.

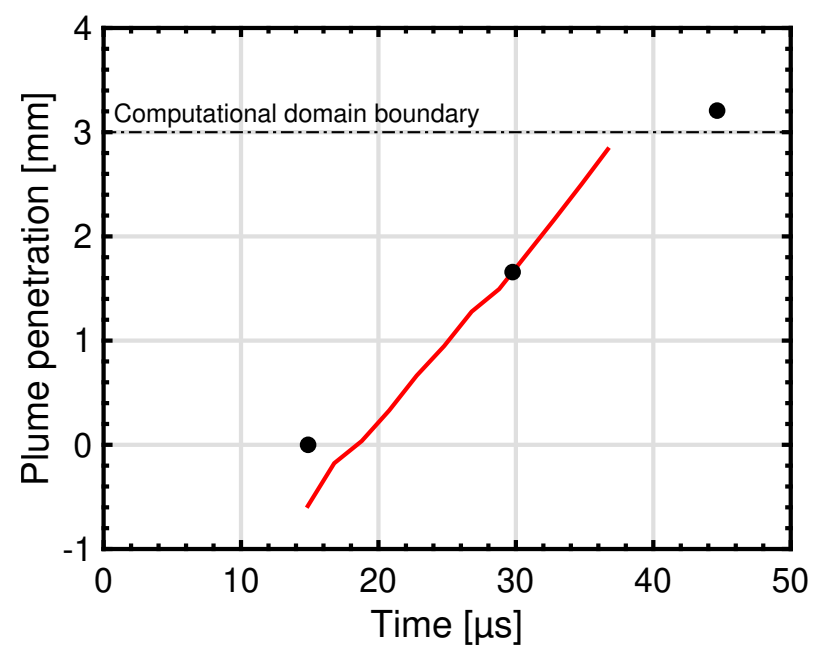

Figure 2. Validation comparison between data collected by Sandia National Laboratories [21] and the VOF-LES 3-Phase method presented. Plume penetration $=0 \mathrm{~mm}$ is the injector nozzle tip, VOF-LES 3-phase measurements taken from nozzle exit (Plume penetration $=-0.661 \mathrm{~mm}$ ). Time $=0 \mu$ s is SOI from the experimental works [21]

\section{Results and discussion}

Even though in GDI injectors the jet velocities and injection pressures are not as high as in diesel injection still they are high enough that can cause pressure driven effects (cavitation, hydraulic flip). In this section we will investigate how these phenomena develop within the nozzle and how they affect the near-region flow pattern and subsequent break-up.

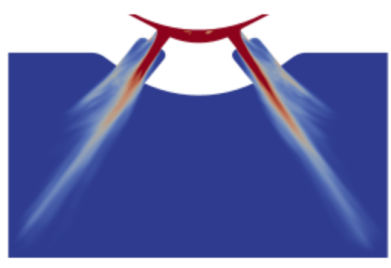

a) $\bar{\alpha}: \# 1, \# 5$

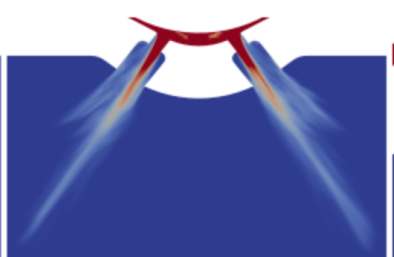

b) $\bar{\alpha}: \# 3, \# 7$

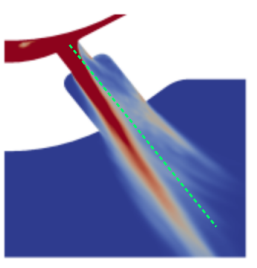

c) $\bar{\alpha}: \# 5$

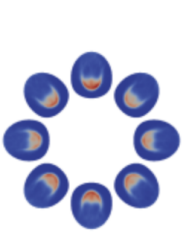

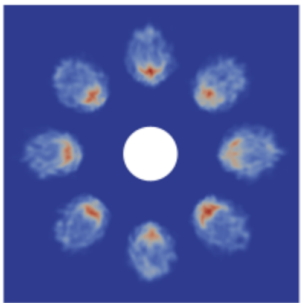

e) $z=0.5 \mathrm{~mm}$

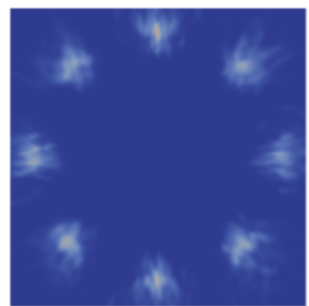

f) $z=1.0 \mathrm{~mm}$

d) $\quad z=0.0 \mathrm{~mm}$
0
Mean liquid volume fraction, $\bar{\alpha}$
0.25

Figure 3. Colour Distribution of the mean liquid volume fraction take over a total time of $\mathrm{t}_{\text {total }}=20 \mu \mathrm{s}$. The slices were taken across the centre of nozzles \#1-\#5, and \#3-\#7. 
Figure 2 shows comparisons of the plume penetration from simulation with experimental data from Sandia National Laboratories [21]. The plume penetration in Figure 2 is taken as the point in which the liquid volume fraction $\alpha \geq 0.1$. The presented numerical data starts at the instant that the liquid exits from the counter-bore, with the penetration slightly over-predicted for the liquid length, this corresponds to numerical results seen with previously [22].

Figure 3 presents the average liquid volume fraction distribution. Typically for single hole sprays the spray angle follows the drill angle of the nozzle (dashed green line). However here, the spray angle is directed slightly inwards. Experimentally this angle at $20 \mu \mathrm{s}$ was found to be $32.3^{\circ}$ from the $z$ axis [5], which should be taken with caution due to potential experimental limitations [21], and in our case this averaged to $33.7^{\circ}$ which represents a $3.3^{\circ}$ deviation from the nozzle direction. This inward pointing of the plumes increases the likelihood of spray collapse between the different plumes, as also showed by Duke et. al [18]. It should be noted that this is the angle of the dense core of the liquid. The average distribution also shows liquid structures of lower density leaving the full radius of the counter-bore hole. This is evident in Figure 5, which occurs from the interaction of trapped nitrogen in the sac of the injector and the initial onset of cavitation inside the nozzle at the inlet.

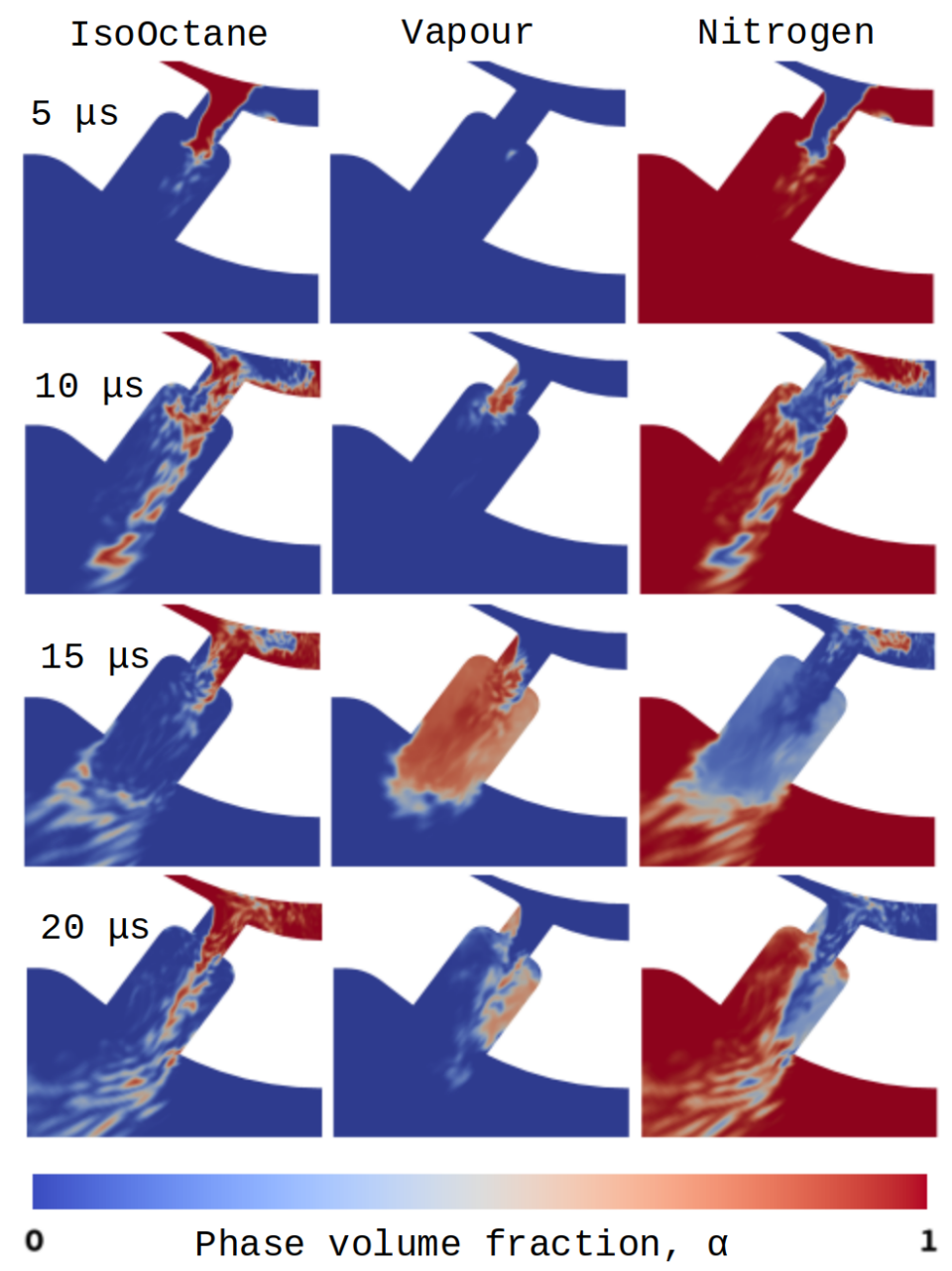

Figure 4. Instantaneous volume fraction colour distributions across a slice taken at nozzle \#1 over four different time steps: 5, 10, 15, and $20 \mu$ s. Red indicates that the volume is filled with the given phase and blue indicates the absence of it.

The transient interaction of the three transported fluids, more specifically gaseous vapour and nitrogen, is more clearly demonstrated in Figure 4. The colour distributions of the three transported fluids has been extracted from a central slice over four time steps. At $t=5 \mu s$ we can see that the initial flow of the iso-octane causes a pocket of nitrogen to be trapped within the 
injector sac. Approximately at this point the initial onset of cavitation can be observed though a consistent cavity structure has yet to form. As the trapped nitrogen is compressed it is forced towards the nozzles and by $t=10 \mu \mathrm{s}$, the nitrogen is now mixing with the main flow. The lower density of the bubbly liquid-nitrogen mixture causes the flow through the nozzle to accelerate to maintain mass flow. This is a catalyst for a consistent wall attached vapour cavity forming from the upper vertex of the nozzle inlet. Thus there is a bubbly mixture of liquid-vapour at the upper wall of the nozzle and liquid-nitrogen at the bottom wall. The liquid structures at the initial part of the spray which didn't mix with the nitrogen propagates towards the exit of the counter-bore hole forming a liquid interface across the radius of the counter-bore, the momentum of which generates a drop in pressure upstream. By $t=15 \mu \mathrm{s}$, this pressure drop causes the iso-octane to completely vaporize within the counter bore momentarily. This large region of vapour created mixes with the nitrogen, whilst the compressed interface of the liquid is still maintained. Diffusive mixing is enabled between these two phases and a largely homogeneous mixture can be observed within the counter-bore hole region except near the jet core. At $t=20$ $\mu s$ the initial spray structures are downstream to the counter-bore outlet and the nitrogen has already started to re-circulate back into the counter bore. This coincides with an increase of local pressure within the counter-bore thus the iso-octane starts to partially condense back to liquid structures. A new cavity structure forms at the lower wall of the counter-bore inlet and homogeneous diffusive mixing continues between the nitrogen and vapour in both cavitation regions. It should be noted that the numerical framework employed here is incompressible, so the velocity changes due to the vapour and nitrogen mixtures forming inside the nozzle will be less severe. Also, a pressure gradient is applied at the inlet of the numerical grid and does not result from a moving needle. Thus the accuracy of the formed structures of trapped nitrogen within the body of the injector is unknown. Regardless, here it has been shown that trapped gaseous structures could trigger non-linear effects to the flow within the short nozzle and counter-bore geometry.

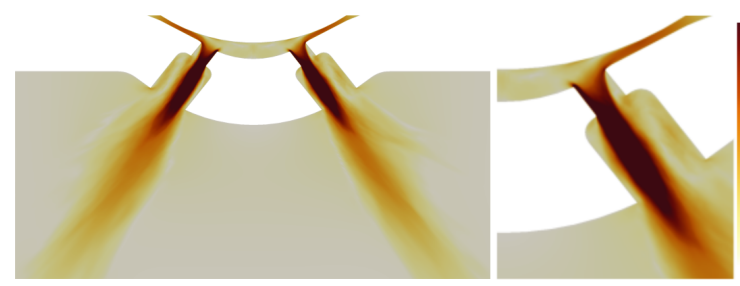

a) b) $\overline{\mathbf{u}}: \# 5$

e) $\quad z=0.5 \mathrm{~mm}$

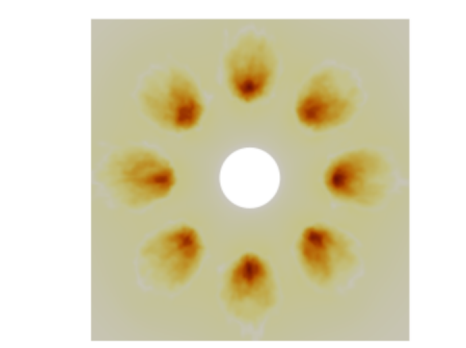

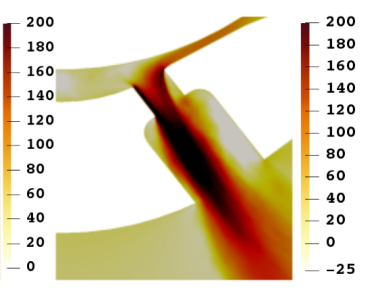

c) $\bar{u}_{z}: \# 5$

d) $\quad z=0.0 \mathrm{~mm}$
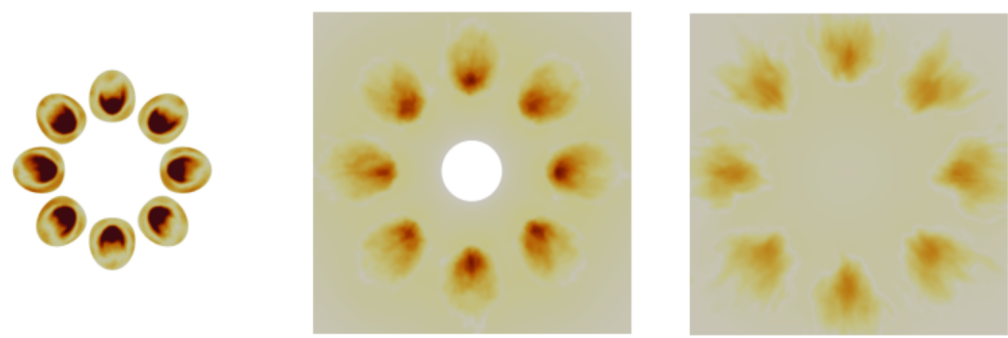

f) $z=1.0 \mathrm{~mm}$

$\begin{array}{lllllll}40 & 60 & 80 & 100 & 120 & 140 & 160\end{array}$ $\overline{\mathrm{u}}\left(\mathrm{ms}^{-1}\right)$

Figure 5. Distribution of the mean velocity take over a total time of $\mathrm{t}_{\text {total }}=20 \mu \mathrm{m}$. The slices were taken across the centre of nozzles \#1-\#5.

The average velocity across the centre of nozzles \#1-\#5 is presented in Figure 5 . The recirculation along the upper wall of the counter-bore is evident with the z-component distribution shown in section c). The increase in velocity of the liquid core within the nozzle maintains its momentum within the counter-bore. This is due to the re-circulating gases even-though the 
flow area is larger at this point. The z-plane slices, sections d), e), ad f), show the high velocity of the liquid core biasing towards the lower/inner wall of the nozzle. The small liquid structures that were formed due to trapped nitrogen being forced through the nozzle can be seen from the lower velocities away from the centre of the main flow downstream from the nozzle. This is highlighted in Figure 6, presenting the iso-surface of the liquid volume fraction $\alpha=0.1$ from the side and bottom view of the injector. Downstream from the nozzle it can be see from the bottom view that the structures from the liquid core maintain a velocity of approximately 150 $\mathrm{m} / \mathrm{s}$, whereas the structures that have come from the upper wall of the counter-bore, seen from the side view, have a much lower velocity.

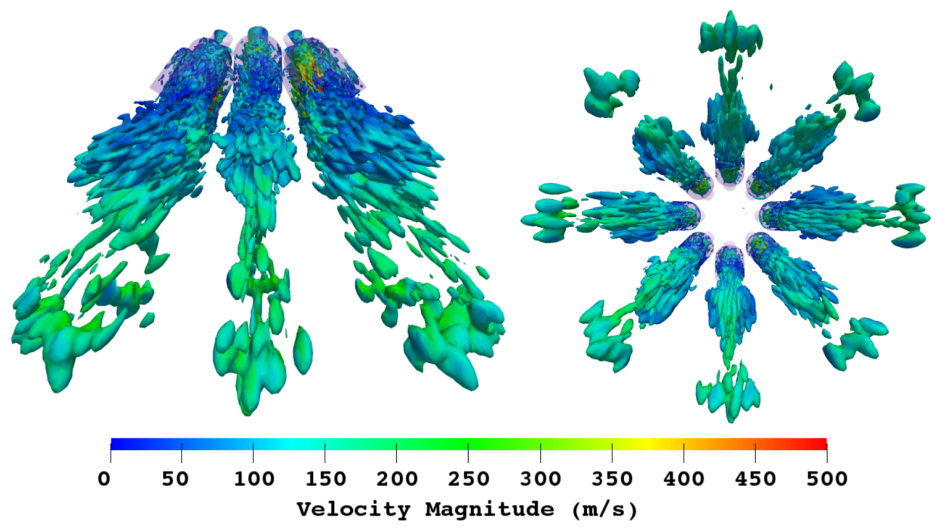

Figure 6. Iso-contours of the surface of the liquid volume fraction at $\alpha=0.1$. The colour distribution imposed on the iso-contours indicates the velocity magnitude of that surface.

\section{Conclusions}

In this study, a novel three fluid VoF spray modelling methodology has been developed and used in order to model the internal nozzle flow coupled with the external GDI multihole spray systems (ECN Spray G). The cavitation formation on a multi-hole non-symmetric configuration has been analysed. A pressure gradient constant in time has been applied at the inlet, focusing the investigation only on the asymmetry imposed by the geometry, leaving the analysis of the needle lift in the future. The presence of cavitation is observed from the interaction of the fuel jet with the nozzle wall. The onset of the cavitation at this point results in a vapour formation along the nozzle and part of the counter-bore, allowing significant upstream penetration of gas in nearly all holes. Trapped nitrogen gas produced non-linear effects and significant vapour and nitrogen diffusive mixing. The asymmetry of the geometry is reflected on the cavitation as the vapour penetration is not equal across the different injector holes. Overall, the method is able to reproduce the cavitation phenomena in the multi-hole configuration, showing the influence of the geometry on the vapour formation mechanism. The future investigation will cover the influence of the needle lift on the cavitation mechanisms and the interactions of the different holes among each other.

\section{Acknowledgements}

This work was funded by the UK's Engineering and Physical Science Research Council [grants EP/S001824/1, Studentship 1990670, iCASE Studentship 2295772].

\section{Nomenclature}

$\begin{array}{ll}\alpha & \text { Phase volume fraction } \\ \rho & \text { Density }\left[\mathrm{kg} / \mathrm{m}^{3}\right] \\ U & \text { Velocity }[\mathrm{m} / \mathrm{s}]\end{array}$


$\begin{array}{ll}m & \text { Mass }[\mathrm{kg}] \\ \Gamma & \text { Diffusion coefficient } \\ C & \text { Rate of phase change coefficient } \\ R_{b} & \text { Instantaneous bubble radius [m] }\end{array}$

\section{References}

[1] Advance Propulsion Centre UK, Light Duty Vehicle Roadmap 2020, Feb. 2021, Available at: https://www.apcuk.co.uk/product-roadmaps/ [Accessed 23rd Feb. 2021]

[2] Wang, C., Xu, H., Herreros, JM.,Wang, J., Cracknell, R., 2014, Applied Energy, 132, pp. 178-191.

[3] Li, X., Cheng, Y., Ji, S., Yang, X., Wang, L., 2019, Energies, 12, pp. 434.

[4] Saha, K., Som, S., Battistoni, M., Li, Y., Pomraning, E., Senecal, P., K., 2016, SAE Int. J. Engines, 9, pp. 1230-1240.

[5] Spray G ECN, Sandia National Laboratory, ecn.sandia.gov

[6] Allocca, L., Bartolucci, L., Cordiner, S., Lazzaro, M., Montanaro, A., Mulone, V., Rocco, V., Apr. 2018, WCX World Congress Experience.

[7] Strek, P., Duke, D., Swantek, A., Kastengren, A., Powell, C., F., Schmidt, D., P., Apr. 2016, SAE 2016 World Congress and Exhibition.

[8] Saha, K., Quan, S., Battistoni, M., Som, S., Senecal, P., K., Pomraning, E., 2017, SAE Technical Paper Series, 1.

[9] Saha, K., Srivastava, P., Quan, S., Senecal, P., K., Pomraning, E., Som, S., Apr. 2018, WCX World Congress Experience.

[10] Montanaro, A., Allocca, L., Sep. 2015, JSAE/SAE 2015 International Powertrains, Fuels \& Lubricants Meeting.

[11] Montanaro, A., Allocca, L., Lazzaro, M., Oct. 2017, International Powertrains, Fuels \& Lubricants Meeting.

[12] Manin, J., Jung, Y., Skeen, S., A., Pickett, L., M., Parrish, S., E., Markle, L., Sep. 2015, JSAE/SAE 2015 International Powertrains, Fuels \& Lubricants Meeting.

[13] Baldwin, E., T., Grover, R., O., Parrish, S., E., Duke, D., J., Matusik, K., E., Powell, C., F., Kastengren, A., L., Schmidt, D., P., 2016, Int. J. of Multiphase Flow, 87, pp. 90-101.

[14] Tahmasebi, E., 2016, "Simulation of Internal Flow in Fuel Injection Process". Politecnico Di Milano.

[15] Jasak, H., Jemcov, A., Tukovic, Z., et al., 2007, International workshop on coupled methods in numerical dynamics.

[16] Sauer, J., Schnerr, G., H., Jun. 2000, ASME Fluids Engineering Summer Conference.

[17] Lemmon, E., W., McLindem, M., O., Friend, D., G., 2021 Thermophysical Properties of Fluid Systems in NIST Chemistry WebBook, NIST Standard Reference Database Number 69.

[18] Duke, D., Kastengren, A., Matusik, K., Swantek, A., Powell, C., Payri, R., Vaquerizo, D., Itani, L., Bruneaux, G., Grover Jr., R., Parrish, S., Markle, L., Schmidt, D., Manin, J., Skeen, S., Pickett, L., 2017, Experimental Thermal and Fluid Science, 88, pp. 608-621.

[19] McGinn, P., Vogiatzaki, K., May. 19.-24. 2019, 10th International Conference on Multiphase Flow.

[20] Nicoud, F., Ducros, F., 1999, Flow, Turbulence and Combustion.

[21] Hwang, J., Weiss, L., Karathanassis, I.K., Koukouvinis, P., Pickett, L.M. and Skeen, S.A., 2020. Fuel, 280, p.118359.

[22] Navarro-Martinez, S., Tretola, G., Reza Yosri, M., Gordon, R.L. and Vogiatzaki, K. 2020, Int. J. Engine Res, 1, pp 217-225. 